\title{
Safety and efficacy of intravenous infusion of allogeneic cryopreserved mesenchymal stem cells for treatment of chronic kidney disease in cats: results of three sequential pilot studies
}

\author{
Jessica M Quimby ${ }^{1 *}$, Tracy L Webb ${ }^{1}$, Lauren M Habenicht ${ }^{1}$ and Steven W Dow ${ }^{1,2}$
}

\begin{abstract}
Introduction: Administration of mesenchymal stem cells (MSCs) has been shown to improve renal function in rodent models of chronic kidney disease (CKD), in part by reducing intrarenal inflammation and suppressing fibrosis. CKD in cats is characterized by tubulointerstitial inflammation and fibrosis, and thus treatment with MSCs might improve renal function and urinary markers of inflammation in this disease. Therefore, a series of pilot studies was conducted to assess the safety and efficacy of intravenous administration of allogeneic adipose-derived MSCS (aMSCs) in cats with naturally occurring CKD.

Methods: Cats enrolled in these studies received an intravenous infusion of allogeneic aMSCs every 2 weeks collected from healthy, young, specific pathogen-free cats. Cats in pilot study 1 (six cats) received $2 \times 10^{6}$ cryopreserved aMSCs per infusion, cats in pilot study 2 (five cats) received $4 \times 10^{6}$ cryopreserved aMSCs per infusion, and cats in pilot study 3 (five cats) received $4 \times 10^{6}$ aMSCs cultured from cryopreserved adipose. Serum biochemistry, complete blood count, urinalysis, urine protein, glomerular filtration rate, and urinary cytokine concentrations were monitored during the treatment period. Changes in clinical parameters were compared statistically by means of repeated measures analysis of variance (ANOVA) followed by Bonferroni's correction.

Results: Cats in pilot study 1 had few adverse effects from the aMSC infusions and there was a statistically significant decrease in serum creatinine concentrations during the study period, however the degree of decrease seems unlikely to be clinically relevant. Adverse effects of the aMSC infusion in cats in pilot study 2 included vomiting (2/5 cats) during infusion and increased respiratory rate and effort (4/5 cats). Cats in pilot study 3 did not experience any adverse side effects. Serum creatinine concentrations and glomerular filtration rates did not change significantly in cats in pilot studies 2 and 3.

Conclusions: Administration of cryopreserved aMSCs was associated with significant adverse effects and no discernible clinically relevant improvement in renal functional parameters. Administration of aMSCs cultured from cryopreserved adipose was not associated with adverse effects, but was also not associated with improvement in renal functional parameters.
\end{abstract}

Keywords: Cell culture, Cytokines, Feline, Kidney, Mesenchymal stem cells, Urine

\footnotetext{
* Correspondence: jquimby@colostate.edu

${ }^{1}$ Department of Clinical Sciences, Immunology, and Pathology, Center for Immune and Regenerative Medicine, College of Veterinary Medicine and Biomedical Sciences, Colorado State University, Fort Collins, CO 80523, USA Full list of author information is available at the end of the article
} 


\section{Introduction}

Chronic kidney disease (CKD) is a common condition in elderly cats and is characterized by tubulointerstitial inflammation, tubular atrophy and interstitial fibrosis with subsequent progressive loss of renal function $[1,2]$. Currently there is no definitive therapy short of renal transplant to improve kidney function in cats with CKD. Therefore, novel and effective therapeutic options are highly desired for treating this disease in cats.

Recently, a number of studies have suggested the use of mesenchymal stem cells (MSCs) as a novel treatment option for management of CKD, based on encouraging data from rodent studies [3-7]. There have been several rationales advanced for the use of MSCs for treatment of CKD. Rodent studies have suggested that MSCs may incorporate into the renal parenchyma and give rise to new renal tubular cells, though the process appears to be relatively inefficient [8-17]. MSCs also exert potent anti-inflammatory and antifibrotic effects and may therefore indirectly improve renal function by reducing diseaseassociated inflammation and fibrosis through paracrine effects [3-7]. For example, MSCs have been shown to inhibit lymphocyte proliferation and cytokine production, suppress dendritic cell function, and suppress interferon $\gamma$ (IFNY) production by natural killer (NK) cells [18].

Since inflammation appears to be present at all stages of CKD in cats, the immunomodulatory actions of MSCs are appealing as an alternative means of suppressing intrarenal inflammation long term and with fewer side effects than with conventional anti-inflammatory drugs. In the majority of experimentally-induced CKD models investigated, MSC administration resulted in improved renal function, decreased intrarenal inflammation, and reduction of renal fibrosis [3-7]. Thus, MSC therapy may be an effective new approach to slow the progression of CKD and improve renal function.

Previous studies have demonstrated that cultured MSCs can be administered intravenously to rodents as well as to humans. However, in rodent models there is a significant risk of pulmonary thrombosis when high numbers of MSCs are rapidly administered intravenously [19]. Alternative routes of delivery have included injection via the renal artery, injection directly into the renal parenchyma, and injection into the renal subcapsular space [3-7]. One potential advantage of intravenous delivery compared to other routes may be the induction of renotropic paracrine effects following intravenous administration of MSCs [20].

Based on compelling results from rodent studies, we conducted a series of pilot studies to investigate the safety and potential efficacy of adipose-derived MSC (aMSC) therapy as a treatment for cats with naturally occurring CKD. These studies were designed to test the hypothesis that allogeneic cryopreserved aMSCs could be safely administered to cats with CKD and would result in improvement in kidney function. For this study, aMSCs were derived from allogeneic, healthy, specific pathogen-free (SPF) donor cats and cryopreserved, either as cells or adipose tissue, prior to intravenous administration to study cats. One primary study endpoint was to determine safety and potential adverse effects of repeated intravenous administration of cryopreserved aMSCs. The second major endpoint was to determine whether repeated MSC administrations were associated with improvement in renal function or urinary markers of intrarenal inflammation. These studies produced evidence of modest, but unlikely to be clinically significant, improvement in renal function but also showed evidence of significant adverse effects associated with intravenous administration of higher doses of cryopreserved aMSCs.

\section{Methods}

\section{Study cats}

Cats with stable CKD, serum creatinine 1.6 to $5.0 \mathrm{mg} / \mathrm{dl}$, were recruited from the patient population at the Veterinary Teaching Hospital at Colorado State University. Cats were determined to have stable CKD based on two repeated biochemical evaluations performed at least 2 weeks apart and ultrasonographic evidence of CKD. Pretreatment evaluation included complete blood count $(\mathrm{CBC})$, biochemistry profile, urinalysis, urine culture, blood pressure, total $\mathrm{T} 4$, urine protein creatinine ratio (UPC), feline leukemia/feline immunodeficiency virus serology, and a renal ultrasound. Cats were excluded from the study if they had evidence of ureteroliths, pyelonephritis, anatomic abnormalities such as polycystic kidney disease or masses, uncontrolled hypertension, or concurrent systemic disease. Administration of concurrent supportive therapies was allowed provided there were no changes in therapy during the study period. The study was approved by the Institutional Animal Care and Use Committee at Colorado State University (\#10$1603 \mathrm{~A}$ and 11-2915A), and all owners reviewed and signed consent forms prior to participation in the study.

\section{aMSC preparation and administration: pilot studies 1 and 2}

MSCs were isolated from adipose tissue of donor SPF cats obtained from the same subcutaneous site on the ventral abdomen just caudal to the umbilicus, as previously described $[21,22]$. For isolation of the stromal vascular fraction, the tissue was minced and digested with $1 \mathrm{mg} / \mathrm{ml}$ collagenase (Sigma Aldrich, St Louis, MO, USA) for $30 \mathrm{mi}-$ nutes at $37^{\circ} \mathrm{C}$. The sample was centrifuged, and the stromal vascular fraction was plated in MSC medium (low glucose Dulbecco's modified Eagle medium (DMEM), $100 \mathrm{U} / \mathrm{ml}$ penicillin, $100 \mu \mathrm{g} / \mathrm{ml}$ streptomycin, $2 \mu \mathrm{M}$ L-glutamine, $1 \%$ essential amino acids without L-glutamine, $1 \%$ nonessential amino acids, $0.075 \%$ sodium bicarbonate (Invitrogen/ Gibco, Carlsbad, CA, USA) plus 15\% fetal bovine serum 
(Cell Generation, Fort Collins, CO, USA)). The aMSCs were incubated until approximately $70 \%$ confluent with media changes every 2 to 3 days. After reaching passage 3 , cells were harvested, divided into treatment aliquots in freezing medium (11\% dimethyl sulfoxide, $14 \%$ MSC medium, $75 \%$ fetal bovine serum), and stored in liquid nitrogen for no greater than 1 year prior to use. For injection in pilot studies 1 and 2, cryopreserved cells were removed from liquid nitrogen, incubated in MSC medium for 10 minutes, washed three times in Dulbecco's phosphate buffered saline (DPBS; Sigma) and then counted and checked for viability using Trypan Blue (cells with less than $95 \%$ viability were not used). For cats in pilot study 1 , $2 \times 10^{6}$ cells were resuspended in $5 \mathrm{ml}$ Hank's balanced salt solution (HBSS) with 200 IU heparin sulfate and administered slowly by hand over 15 minutes; aMSCs were administered at weeks 0,2 and 4. For cats in pilot study 2, $4 \times 10^{6}$ cells were initially resuspended in $10 \mathrm{ml}$ HBSS with 200 IU heparin sulfate and administered as a slow intravenous push over 30 minutes. However, the first cat to receive MSCs in this manner experienced an increased respiratory rate. Subsequently, cells for the high-dose group were resuspended in $20 \mathrm{ml} \mathrm{HBSS} / 200 \mathrm{IU}$ heparin and administered over 45 to 60 minutes with a syringe pump with frequent agitation of the syringe to prevent settling of the cells. Cats in pilot study 2 received aMSCs at weeks 2,4 , and 6 . All three injections received by cats treated in pilot studies 1 and 2 were from the same donor cat. A total of four donor cats were used to provide aMSCs for those studies.

\section{MSC preparation and administration: pilot study 3}

MSCs were isolated from adipose tissue of donor SPF cats as described above. For preparation of the adipose tissue for cryopreservation, the tissue was minced and divided into 1 -g aliquots in $1 \mathrm{ml}$ of freezing medium $(11 \%$ dimethyl sulfoxide, $14 \%$ MSC medium, $75 \%$ fetal bovine serum), and stored in liquid nitrogen for no greater than 1 year prior to use. Cryopreserved adipose was then later thawed, immediately washed twice with DPBS, and then prepared for culture as described above. For injection in pilot study 3 cats, cells were harvested by trypsinization at passage 3 and resuspended in $10 \mathrm{ml}$ DPBS with $200 \mathrm{IU}$ heparin sulfate and administered as a slow intravenous push over 20 minutes. Cats in pilot study 3 received aMSCs at weeks 2, 4, and 6 . All three injections received by cats treated in pilot study 3 were from different donor cats given in a prerandomized order. A total of three donor cats were used to provide aMSCs for the study.

\section{Characterization of MSCs}

aMSCs cultured from cryopreserved adipose tissue were characterized by surface marker expression using flow cytometry and a panel of crossreactive antibodies specific for surface determinants expressed by MSCs from other species [23-26]. Specifically, feline aMSCs were analyzed for surface expression of CD44 (antimouse/human, antibody clone:IM7, eBioscience, San Diego, CA, USA) and CD90 (anti-human, antibody clone:eBio5E10, eBioscience). MSCs were also assessed for expression of CD4 (anti-feline antibody clone: 3-4F4, Southern Biotech, Birmingham, AL, USA) and major histocompatibility complex (MHC) class II (anti-feline antibody clone: TU39, BD Bioscience, San Jose, CA, USA). Samples were analyzed using a Cyan ADP flow cytometer (Beckman Coulter, Brea, CA, USA). Approximately 25,000 events were collected for analysis per sample.

In vitro differentiation assays were conducted to confirm the multipotency of feline aMSCs, as assessed by their ability to differentiate into three cell lineages (osteoblasts, chondrocytes, and adipocytes) that are characteristic of MSCs [27]. For differentiation into adipocytes, aMSCs at confluency were incubated with MSC medium supplemented with $0.5 \mu \mathrm{M}$ dexamethasone (Sigma Aldrich), $50 \mu \mathrm{M}$ indomethacin (Sigma Aldrich) and $0.5 \mu \mathrm{M} 3$ isobutyl-1-methylxantine (Sigma Aldrich) for 3 weeks with media changes every 3 to 4 days. Chondrogenic differentiation medium consisted of DMEM $1 \times$ (Cellgro, Manassas, VA, USA) supplemented with 15\% fetal bovine serum (FBS; Cell Generation), $10 \mathrm{nM}$ dexamethasone (Sigma Aldrich), $10 \mathrm{ng} / \mathrm{ml}$ transforming growth factor (TGF)- $\beta$ (R\&D Systems, Minneapolis, MN, USA), $50 \mu \mathrm{g} / \mathrm{ml}$ ascorbic acid (Sigma Aldrich), and $40 \mu \mathrm{g} / \mathrm{ml}$ proline (Sigma Aldrich). Osteogenic differentiation medium consisted of MSC medium supplemented with $10 \mathrm{nM}$ dexamethasone, $50 \mu \mathrm{M}$ ascorbic acid, and $20 \mathrm{mM} \beta$-glycerophosphate (Sigma Aldrich) At the end of the differentiation period, cells were fixed with $10 \%$ neutral buffered formalin and stained with Oil Red O (Sigma Aldrich) for presence of lipid or with Alizarin Red (Sigma Aldrich) for the presence of calcium [27]. Cell pellets from cartilage differentiation were harvested and placed in OCT Compound (Sakura Finetek USA, Inc., Torrance, CA, USA) and flash frozen prior to staining with toluidine blue (Richard-Allan Scientific, Kalamazoo, MI, USA) for cartilage matrix. aMSCs cultured in MSC medium alone under identical conditions were used as differentiation controls.

\section{Clinical monitoring \\ Pilot study 1}

Each treated cat underwent physical examination, weighing, and routine blood work consisting of $\mathrm{CBC}$, serum biochemistry, urinalysis, and UPC immediately prior to aMSC injection at week 0 , and at week 2,4 .

\section{Pilot study 2}

Each treated cat underwent physical examination, weighing, and routine blood work consisting of $\mathrm{CBC}$, 
serum biochemistry, and urinalysis at weeks $0,2,4,6$ and 8. Each cat had a UPC performed at weeks 0 and 8 . Additionally each cat had a glomerular filtration rate (GFR) estimated by iohexol clearance performed at weeks 0 and 8 . Iohexol clearance has been described as a clinically applicable alternative to estimate GFR and has previously been assessed in cats with reduced renal function [28-30]. For this method, $300 \mathrm{mg} / \mathrm{kg}$ iohexol (Omnipaque, GE Health Incorporated, Princeton, NJ, USA) was administered intravenously, and blood samples were collected at 2, 3, and $4 \mathrm{~h}$ after administration. Analysis is commercially available at the Michigan State University Diagnostic Center for Population and Animal Health. Assessment of GFR variability was performed by enrolling three CKD cats that did not receive MSC therapy but underwent estimation of GFR by iohexol clearance at 0 and 8 weeks.

\section{Pilot study 3}

Each treated cat underwent physical examination, weighing, and routine blood work consisting of $\mathrm{CBC}$, serum biochemistry, and urinalysis at weeks $0,2,4,6$ and 8. Each cat had a UPC performed at weeks 0 and 8. Additionally, each cat had a GFR by nuclear scintigraphy performed at weeks 0 and 8 .

For the scintigraphy procedure, cats were sedated with a standard sedation protocol (ketamine $10 \mathrm{mg} / \mathrm{cat}$ and butorphanol $0.1 \mathrm{mg} / \mathrm{kg}$ intravenously once) at a standard time before the procedure. A single nuclear technician performed all of the procedures for each particular cat. For each procedure $1.0 \mathrm{mCi}$ of Tc $99 \mathrm{~m}$-labeled diethylene triamine penta-acetic acid (DTPA) (Cardinal Health, Dublin, $\mathrm{OH}, \mathrm{USA}$ ) was injected intravenously via a catheter placed in a standard location in each cat. Images were obtained using GE Millennium SPECT system applicable for small animal planar, whole body and single-photon emission computed tomography (SPECT) imaging (GE Healthcare, Waukesha, WI, USA). Three independent radiologists evaluated the GFR data, and a mean GFR value for each kidney as well as a global value was determined.

\section{Urinary cytokine analysis}

Urine cytokine concentrations were determined using commercial ELISA kits, as we have described recently [31]. Briefly, urine concentrations of interleukin (IL)-6, IL-8, and IL-10 were measured using feline specific ELISA kits (R\&D Systems), while monocyte chemoattractant protein 1 (MCP-1) and vascular endothelial growth factor (VEGF) were measured using commercial canine MCP-1 and VEGF kits respectively (R\&D Systems). Urine transforming growth factor $\beta 1$ (TGF- $\beta 1$ ) concentrations were measured using an ELISA kit from Invitrogen (Camarillo, CA, USA).

Urine samples were collected by cystocentesis, and an aliquot of urine was immediately frozen at $-20^{\circ} \mathrm{C}$ and then stored at $-80^{\circ} \mathrm{C}$ for cytokine measurements. Samples were thawed immediately prior to analysis and kept on ice during sample preparation. All samples were centrifuged at $2000 \mathrm{rpm}(350 \mathrm{~g})$ for 5 minutes at $4^{\circ} \mathrm{C}$, and the supernatant was collected for analysis. With one exception, ELISA kits were run according to manufacturer instructions for serum samples. As one exception, urine samples were diluted 5-fold rather than 40-fold for detection of TGF- $\beta 1$.

Urine and serum creatinine concentrations were measured with a Roche Cobas Integra Chemistry Analyzer (Roche Diagnostics Limited, West Sussex, UK) at the Colorado State University Diagnostic Laboratory. All reported cytokine concentrations were normalized for differences in urine concentration as a urine cytokine-to -urine creatinine ratio. In order to maximize cytokine yield in the urine samples, additional sample processing steps were performed as previously described prior to running the IL-8, MCP-1, and VEGF ELISA kits [31].

\section{Statistical analysis}

Changes in GFR, serum creatinine, blood urea nitrogen (BUN), electrolytes, urine protein-to-creatinine (UPC) ratio, urine specific gravity, and packed cell volume (PCV) data over time in the aMSC-injected cats were compared statistically by means of repeated measures analysis of variance (ANOVA) followed by Bonferroni's correction. Values were considered statistically different for $P<0.05$. Statistical analyses were performed using Prism5 software (GraphPad, La Jolla, CA, USA).

\section{Results}

\section{Description of study cats}

A total of 165 cats with CKD were screened for entry into this series of pilot studies. In all, 52 cats were excluded for geographical reasons (extensive travel required), 40 cats were excluded due to concurrent illness, 18 cats were excluded due to end-stage disease, 13 cats were excluded as the owners thought the study too complex, 11 cats were excluded due to unstable disease, 8 cats were excluded for anatomic abnormalities, and 2 cats were excluded due to fractious attitude in hospital. Descriptive data for the 21 cats with CKD that were enrolled in the aMSC pilot studies are presented in Table 1. Two cats that were enrolled in pilot study 3 were unable to finish the trial due to development of unrelated illnesses. A summary of cat weights throughout the study and aMSC dose by weight are presented in Table 2 . No statistically significant difference in weight was seen in any pilot study as determined by a Wilcoxon sign rank test.

\section{Characterization of MSCs}

During in vitro culture feline aMSCs were observed to develop into a relatively homogeneous population of 
Table 1 Summary of demographics of cats participating in the intravenous allogeneic cryopreserved studies

\begin{tabular}{|c|c|c|c|c|}
\hline Cat no. & Group & Description & IRIS CKD stage and creatinine (mg/dl) & Treatment \\
\hline 1 & Pilot study 1 & 10 yr MC DSH & IRIS CKD II; creatinine: 2.5 & $2 \times 10^{6}$ MSCs intravenously $\times 3$ treatments \\
\hline 2 & Pilot study 1 & 15 yr FS DSH & IRIS CKD III; creatinine: 3.5 & $2 \times 10^{6}$ MSCs intravenously $\times 3$ treatments \\
\hline 3 & Pilot study 1 & 7 yr MC Siamese & IRIS CKD III; creatinine: 4.3 & $2 \times 10^{6}$ MSCs intravenously $\times 3$ treatments \\
\hline 4 & Pilot study 1 & 12 yr MC DLH & IRIS CKD II; creatinine: 2.4 & $2 \times 10^{6}$ MSCs intravenously $\times 3$ treatments \\
\hline 5 & Pilot study 1 & 15 yr MC DSH & IRIS CKD II; creatinine: 2.3 & $2 \times 10^{6}$ MSCs intravenously $\times 3$ treatments \\
\hline 6 & Pilot study 1 & 15 yr FS Siamese & IRIS CKD III; creatinine: 3.5 & $2 \times 10^{6}$ MSCs intravenously $\times 3$ treatments \\
\hline 7 & Pilot study 2 & 11 yr MC DSH & IRIS CKD II; creatinine: 1.9 & $4 \times 10^{6}$ MSCs intravenously $\times 3$ treatments \\
\hline 8 & Pilot study 2 & 11 yr FS DSH & IRIS CKD II; creatinine: 2.6 & $4 \times 10^{6}$ MSCs intravenously $\times 3$ treatments \\
\hline 9 & Pilot study 2 & 18 yr FS DSH & IRIS CKD II; creatinine: 2.8 & $4 \times 10^{6}$ MSCs intravenously $\times 3$ treatments \\
\hline 10 & Pilot study 2 & 15 yr MC DSH & IRIS CKD II; creatinine: 2.2 & $4 \times 10^{6}$ MSCs intravenously $\times 3$ treatments \\
\hline 11 & Pilot study 2 & 7 yr MC DSH & IRIS CKD III; creatinine: 3.7 & $4 \times 10^{6}$ MSCs intravenously $\times 3$ treatments \\
\hline 12 & Pilot study 2 & 15 yr MC DSH & IRIS CKD II; creatinine: 2.3 & lohexol GFR repeatability only \\
\hline 13 & Pilot study 2 & 16 yr MC DSH & IRIS CKD II; creatinine: 2.1 & Iohexol GFR repeatability only \\
\hline 14 & Pilot study 2 & 16 yr MC DSH & IRIS CKD II; creatinine: 1.8 & lohexol GFR repeatability only \\
\hline 15 & Pilot study 3 & 9 yr MC DSH & IRIS CKD II; creatinine: 2.6 & Enrolled but decompensated before treatment initiated \\
\hline 16 & Pilot study 3 & 15 yr MC Siamese & IRIS CKD II; creatinine: 2.4 & $\begin{array}{l}4 \times 10^{6} \text { MSCs intravenously } \times 2 \text { treatments before } \\
\text { recurrence of previous diabetes }\end{array}$ \\
\hline 17 & Pilot study 3 & 13 yr MC Siamese & IRIS CKD II; creatinine: 2.7 & $4 \times 10^{6}$ MSCs intravenously $\times 3$ treatments \\
\hline 18 & Pilot study 3 & 8 yr MC DSH & IRIS CKD II; creatinine: 1.7 & $4 \times 10^{6}$ MSCs intravenously $\times 3$ treatments \\
\hline 19 & Pilot study 3 & 13 yr MC DSH & IRIS CKD II; creatinine: 2.0 & $4 \times 10^{6}$ MSCs intravenously $\times 3$ treatments \\
\hline 20 & Pilot study 3 & 15 yr MC DLH & IRIS CKD II; creatinine: 2.3 & $4 \times 10^{6}$ MSCs intravenously $\times 3$ treatments \\
\hline 21 & Pilot study 3 & 15 yr FS DSH & IRIS CKD III; creatinine: 3.1 & $4 \times 10^{6}$ MSCs intravenously $\times 3$ treatments \\
\hline
\end{tabular}

Six cats were enrolled in pilot study 1 and received $2 \times 10^{6}$ cryopreserved adipose-derived mesenchymal stem cells (aMSCs) per infusion. In pilot study 2 eight cats were enrolled; five received $4 \times 10^{6}$ cryopreserved aMSCs per infusion, and three cats received only iohexol clearance studies. In pilot study 3 , seven cats were enrolled; five cats received $4 \times 10^{6}$ cryopreserved aMSCs cultured from cryopreserved adipose per infusion; two cats developed unrelated medical conditions and were removed from the trial. CKD, chronic kidney disease; GFR, glomerular filtration rate. MC = male castrated, FS = female spayed, DSH = domestic shorthair, DLH = domestic long hair, IRIS = International Renal Interest Society.

plastic-adherent cells with fibroblast-like morphology. Adipose-derived MSCs expressed high levels of CD44 and CD90 and were negative for expression of CD4 and MHC class II (Figure 1). Both cryopreserved aMSCs and aMSCs cultured from cryopreserved fat were capable of trilineage differentiation (Figure 2).

\section{Short-term responses to MSC injection}

Cats in pilot study 1 treated with three separate intravenous infusions of $2 \times 10^{6}$ cryopreserved aMSCs and cats in pilot study 3 treated with three separate intravenous infusions of $4 \times 10^{6}$ aMSCs cultured from cryopreserved fat tolerated their injections without apparent adverse effects. During the infusions and immediately after, the cats appeared clinically normal and unperturbed. However, cats in pilot study 2, each having received an intravenous infusion of $4 \times 10^{6}$ cryopreserved aMSCs, experienced a number of treatment-related adverse effects. The adverse effects included vomiting (two cats) and increased respiratory rate (four cats). In two cats, vomiting and nausea occurred within 2 minutes of initiating the first aMSC infusion and were not associated with respiratory distress.
Administration of diphenhydramine $(1 \mathrm{mg} / \mathrm{kg}$ subcutaneously) and maropitant ( $1 \mathrm{mg} / \mathrm{kg}$ subcutaneously) resulted in cessation of clinical signs of vomiting and nausea. Affected cats were premedicated with diphenhydramine and maropitant for subsequent treatments, and additional adverse gastrointestinal reactions were not noted. One cat that experienced vomiting at the time of the first injection subsequently developed increased respiratory rate at the time of the third aMSC injection.

Increased respiratory rate and effort following aMSC infusion was not pronounced in three of the four pilot study 2 cats that experienced respiratory signs but were quite pronounced in one cat. Average baseline respiratory rate was 44 breaths/minute (range 36 to 60 ) and average increased respiratory rate was 80 breaths/minute (range 60 to 112 breaths/minute). Increased respiratory rate and effort was generally noted after approximately two-thirds of the aMSCs had been administered (that is, after 30 minutes of infusion) in the three cats with mild respiratory signs. One cat, however, had mildly increased respiratory rate on the first infusion, a normal second infusion, and developed marked overt respiratory distress 
Table 2 Weight and mesenchymal stem cell (MSC) dose for cats participating in the intravenous allogeneic cryopreserved MSC studies

\begin{tabular}{llll}
\hline Study & Stage & Weight in $\mathbf{k g}$, median (range) & MSC dose in cells/kg, median (range) \\
\hline Pilot study $1, n=6$ & Before & $4.8(2.7$ to 5.1$)$ & $4.1 \times 10^{5}\left(3.9 \times 10^{5}\right.$ to $\left.7.4 \times 10^{5}\right)$ \\
Pilot study $2, n=5$ & After & $4.8(2.5$ to 5.2$)$ & $8.3 \times 10^{5}\left(6.1 \times 10^{5}\right.$ to $\left.9.1 \times 10^{5}\right)$ \\
& Before & $4.8(4.4$ to 6.2$)$ & \\
Pilot study $3, n=5$ & After & $4.8(4.2$ to 7.1$)$ & $8.4 \times 10^{5}\left(5.6 \times 10^{5}\right.$ to $\left.1.1 \times 10^{6}\right)$ \\
& Before & $4.8(3.7$ to 7.1$)$ & \\
\hline
\end{tabular}

on the third infusion after approximately one-third of the aMSCs had been administered. The respiratory distress was characterized by a sudden onset of increased respiratory rate that quickly led to nasal flare, open mouth breathing, and an orthopnic stance with head extended and elbows out. The injection was stopped, the cat was moved to a critical care ward and placed in an oxygen chamber, and diphenhydramine $(1 \mathrm{mg} / \mathrm{kg}$ subcutaneously) was administered. After 20 minutes no improvement was noted, and the cat became notably nauseated (foaming at the mouth and subsequently vomiting). Maropitant (1 $\mathrm{mg} / \mathrm{kg}$ subcutaneously) and dexamethasone $(0.05 \mathrm{mg} / \mathrm{kg}$ intravenously) were administered. Clinical signs began to abate within 20 minutes of administration of dexamethasone. Open mouth breathing and orthopnea improved within $1 \mathrm{~h}$. Increased

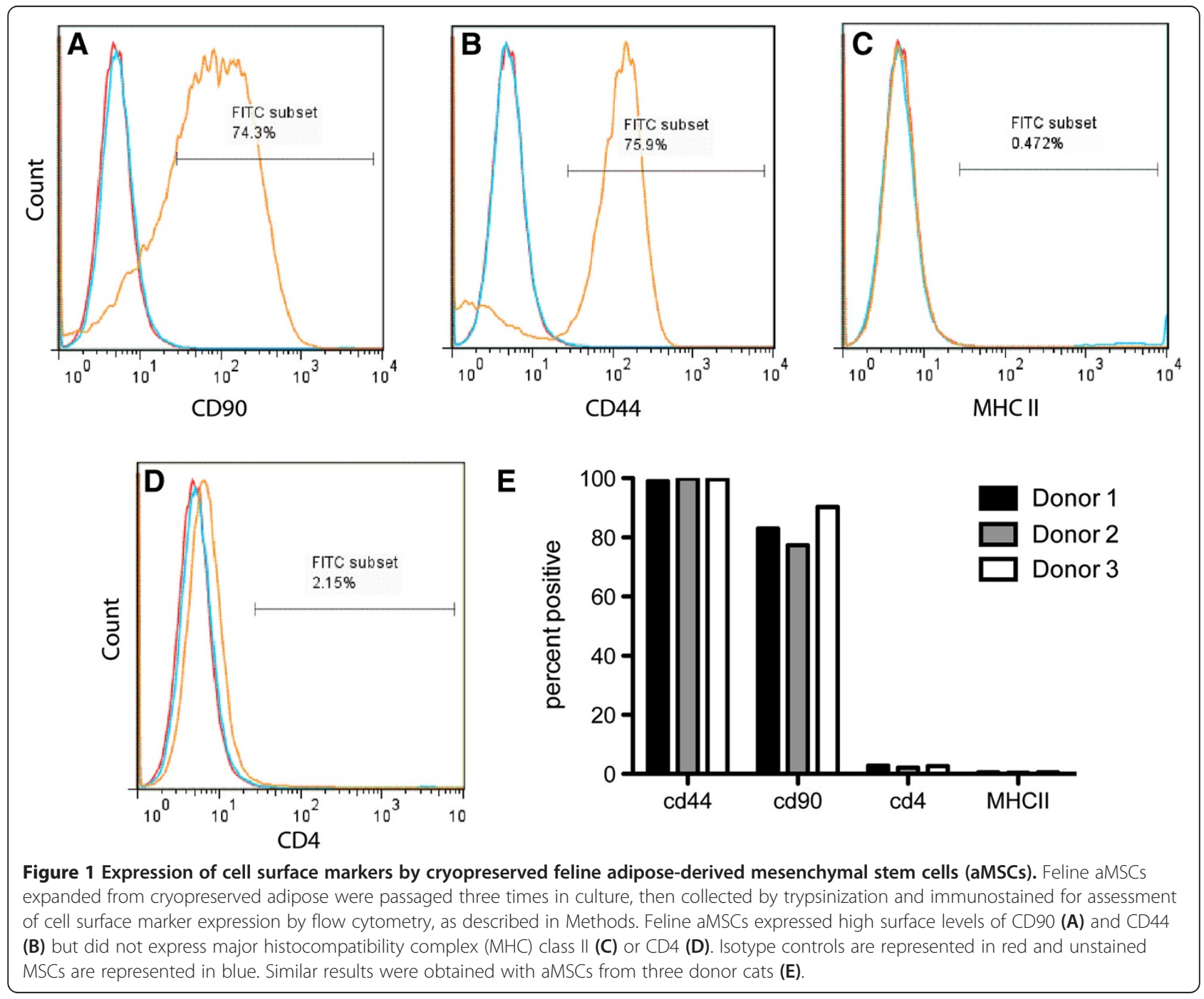



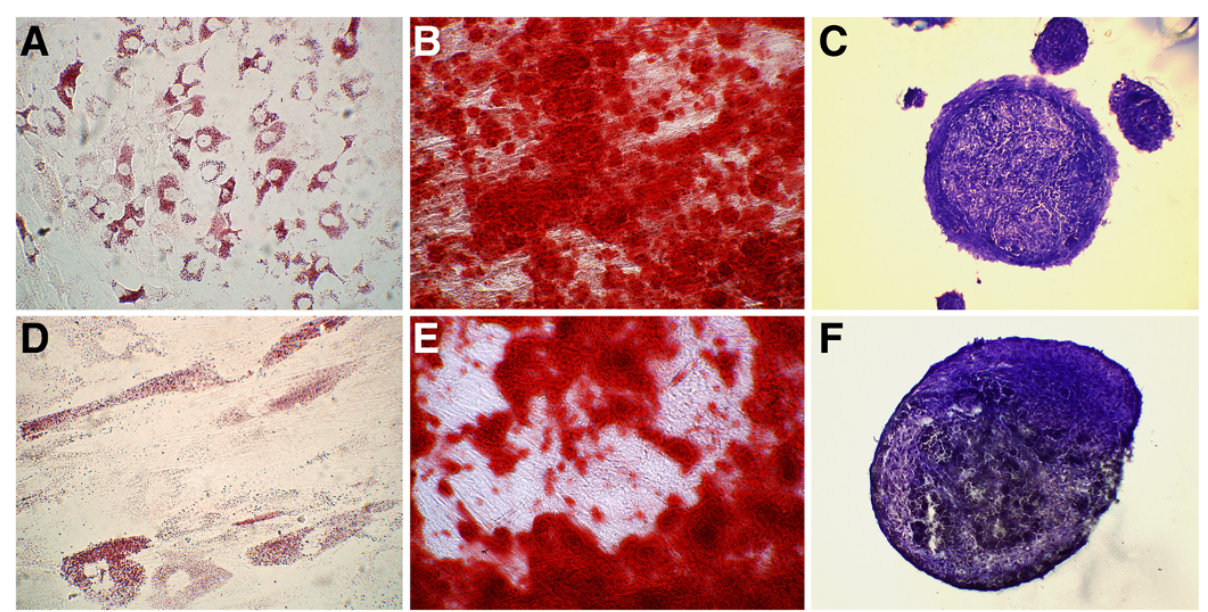

Figure 2 Trilineage differentiation of feline adipose-derived mesenchymal stem cells (aMSCs) after cryopreservation as cells or adipose. aMSCs taken directly from cryopreservation (A-C) and cultured from cryopreserved adipose (D-F) were capable of trilineage differentiation. (A,D) aMSCs formed intracellular lipid vacuoles when incubated in adipocytic differentiation media for 21 days. (B,E) aMSCs stained positive for calcium with alizarin red following differentiation into osteocytic phenotype after 21 days of incubation in differentiation media. (C,F) Cryosection of pellets of cartilage matrix (stained with toluidine blue) formed by aMSCs when exposed to chondrocytic differentiation media for 21 days.

respiratory effort continued for the next $8 \mathrm{~h}$, but by $24 \mathrm{~h}$ post injection the cat was clinically normal and was discharged without further incident.

\section{Effects of MSC administration on renal function clinical parameters}

Serum creatinine concentrations decreased significantly $(P=0.01)$ in pilot study 1 cats over the study period but were unchanged in cats in pilot studies 2 and 3 (Figure 3A-C), however it is our opinion that the degree of observed decrease $(<0.5 \mathrm{mg} / \mathrm{dl})$ in serum creatinine concentrations is of questionable clinical significance. It should be noted that one cat from pilot study 1 was removed from analysis of serum creatinine concentration because the owners failed to administer fluids during the week prior to assessment, in violation of the study protocol. Values for BUN, serum phosphorus, serum potassium, packed cell volume, and urine specific gravity or UPC did not change significantly following administration of aMSCs as compared to pretreatment values in any of the three pilot studies (Table 3).

\section{Effects of aMSC administration on estimated glomerular filtration rate}

The GFR was not measured in pilot study 1 cats. In pilot study 2 cats, there was an overall trend towards improvement in estimated GFR by iohexol clearance values $(P=0.056)$, with individual increases of $(75 \%, 10 \%, 61 \%$, $7 \%, 6 \%)$ compared to untreated CKD control cats $(7 \%,-18 \%$, $-22 \%$ ) (Figure 3D). In pilot study 3 cats there was no statistically significant difference in total or individual kidney GFR values as determined by nuclear scintigraphy however variable individual increases were seen $(63 \%, 7 \%$, $-34 \%, 13 \%, 91 \%$ ) (Figure 3E).

\section{Effects of aMSC administration on urinary cytokine concentrations}

In cats in pilot study 1 , urine MCP-1 and IL- 8 concentration data following MSC administration as measured by ELISA are depicted in Figure 4A,B. Using repeated measures ANOVA, a statistically significant decrease in MCP-1 $(P=0.0001)$ as well as in IL-8 $(P=0.01)$ was detected. However due to the variability in cytokine concentration changes (some cats decreased significantly while others increased), these results may not have clinical relevance.

In cats in pilot studies 2 and 3, urine concentrations of IL-8, MCP-1, TGF- $\beta 1$ and VEGF were assessed (Figure 2C-I). In the case of all four cytokines measured, repeated measures ANOVA failed to detect any significant changes in cytokine concentrations when pretreatment urine cytokine concentrations were compared to samples taken throughout the treatment period. In pilot study 3, MCP-1 levels were inconsistently detected in study samples.

\section{Discussion}

The safety and potential efficacy of repeated intravenous administration of allogeneic cryopreserved feline aMSCs to cats with naturally occurring CKD was assessed in this series of pilot studies. MSCs have previously been shown to be effective in suppressing some aspects of renal disease in rodent models of induced CKD. However, MSC therapy has not been evaluated in a naturally 


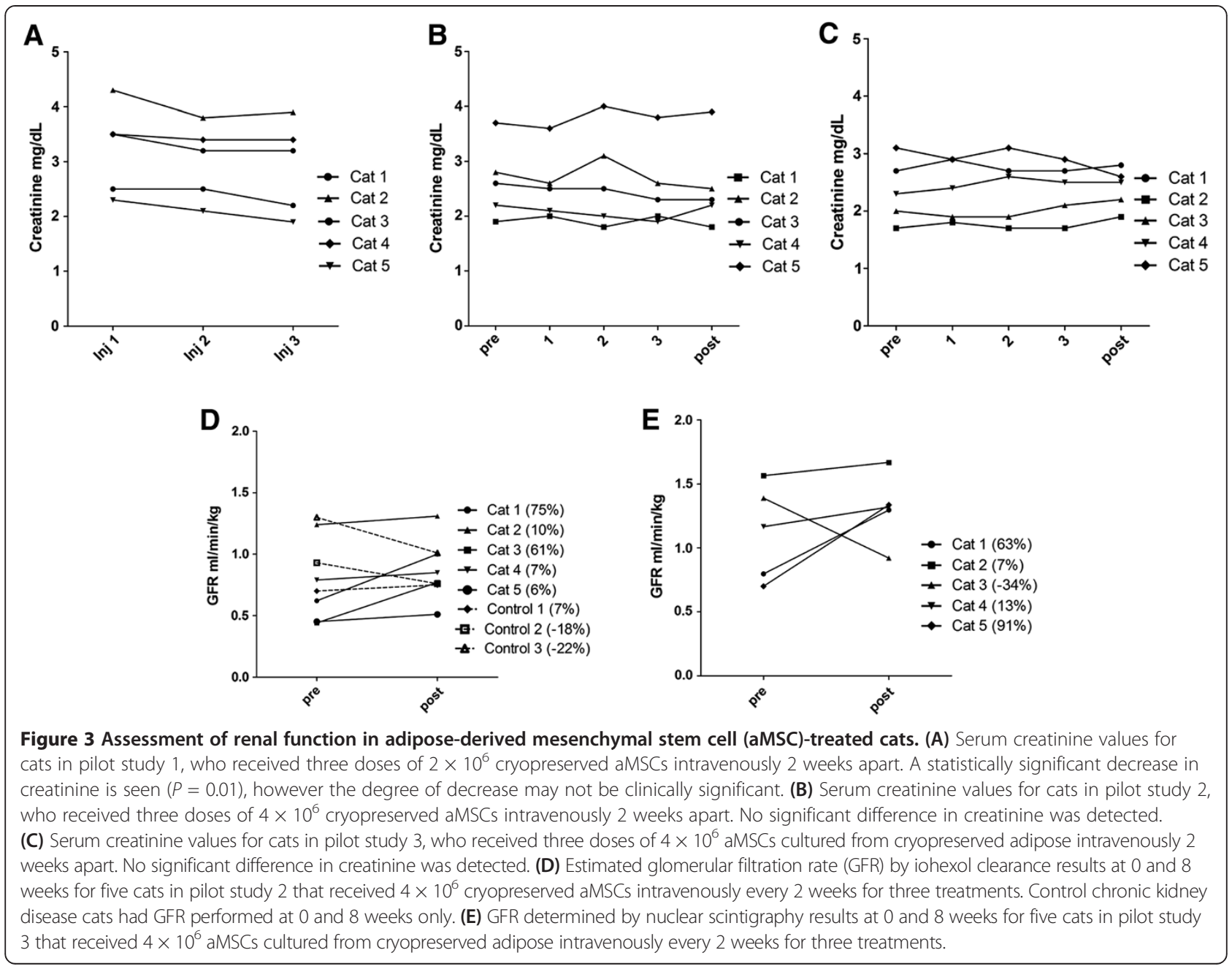

occurring animal model of CKD such as the feline CKD model. We modified existing rodent MSC administration protocols to create a clinically feasible trial in cats with CKD with translational potential. The major findings from our preliminary studies of aMSC therapy were that lower doses of allogeneic cryopreserved aMSCs were well tolerated following repeated intravenous administration and appeared to be associated with modest improvement in renal function. The level of improvement in renal function seen in the treated cats is, however, of uncertain clinical significance. The six cats in pilot study 1 that received the $2 \times 10^{6}$ cryopreserved aMSC dose experienced a statistically significant decrease in serum creatinine together with negligible

Table 3 Summary of clinicopathological data from cats participating in the intravenous allogeneic cryopreserved mesenchymal stem cell (MSC) studies

\begin{tabular}{|c|c|c|c|c|c|c|c|c|}
\hline Study & Stage & $\begin{array}{l}\text { Creatinine } \\
(\mathrm{mg} / \mathrm{dl})\end{array}$ & $\begin{array}{l}\text { Blood urea } \\
\text { nitrogen (mg/dl) }\end{array}$ & $\begin{array}{l}\text { Phosphorus } \\
\text { (mg/dl) }\end{array}$ & $\begin{array}{l}\text { Potassium } \\
\text { (meq/l) }\end{array}$ & $\begin{array}{l}\text { Packed cell } \\
\text { volume }(\%)\end{array}$ & $\begin{array}{l}\text { Urine protein- } \\
\text { to-creatinine } \\
\text { (UPC) ratio }\end{array}$ & $\begin{array}{l}\text { Urine specific } \\
\text { gravity }\end{array}$ \\
\hline \multirow[t]{2}{*}{ Pilot study 1} & Before & $3.5(2.3$ to 4.3$)$ & 57 (33 to 69) & $3.8(2.6$ to 4.5$)$ & 4.6 (3.8 to 5.5$)$ & 31 (29 to 36) & 0.1 (0.1 to 0.2$)$ & $1.016(1.013$ to 1.022$)$ \\
\hline & After & $3.2(1.9$ to 3.9$)$ & 53 (36 to 58 ) & 3.7 (3.3 to 4.5$)$ & $4.2(3.9$ to 4.7$)$ & 28 (26 to 36$)$ & 0.1 (0.1 to 0.2) & $1.016(1.013$ to 1.025$)$ \\
\hline \multirow[t]{2}{*}{ Pilot study 2} & Before & 2.6 (1.9 to 3.7$)$ & 42 (34 to 61$)$ & 4.0 (3.6 to 4.2$)$ & $4.6(3.7$ to 5.1$)$ & 31 (27 to 35$)$ & 0.1 (0.1 to 0.2$)$ & $1.016(1.015$ to 1.030$)$ \\
\hline & After & 2.3 (1.8 to 3.9$)$ & 37 (30 to 64) & 4.7 (2.4 to 6.7$)$ & 4.6 (3.7 to 5.0$)$ & 29 (27 to 34$)$ & 0.1 (0.1 to 0.3$)$ & 1.017 (1.013 to 1.036$)$ \\
\hline \multirow[t]{2}{*}{ Pilot study 3} & Before & $2.3(1.7$ to 3.1$)$ & 49 (34 to 53) & 4.2 (3.6 to 4.9$)$ & 4.7 (3.9 to 5.0$)$ & 34 (25 to 37$)$ & $0.1(0.1$ to 0.4$)$ & 1.017 (1.013 to 1.037$)$ \\
\hline & After & 2.5 (1.9 to 2.8$)$ & 42 (36 to 53$)$ & 3.8 (3.7 to 3.9$)$ & 4.7 (4.1 to 5.0$)$ & 33 (28 to 34$)$ & 0.1 (0.1 to 0.4$)$ & $1.019(1.013$ to 1.029$)$ \\
\hline
\end{tabular}



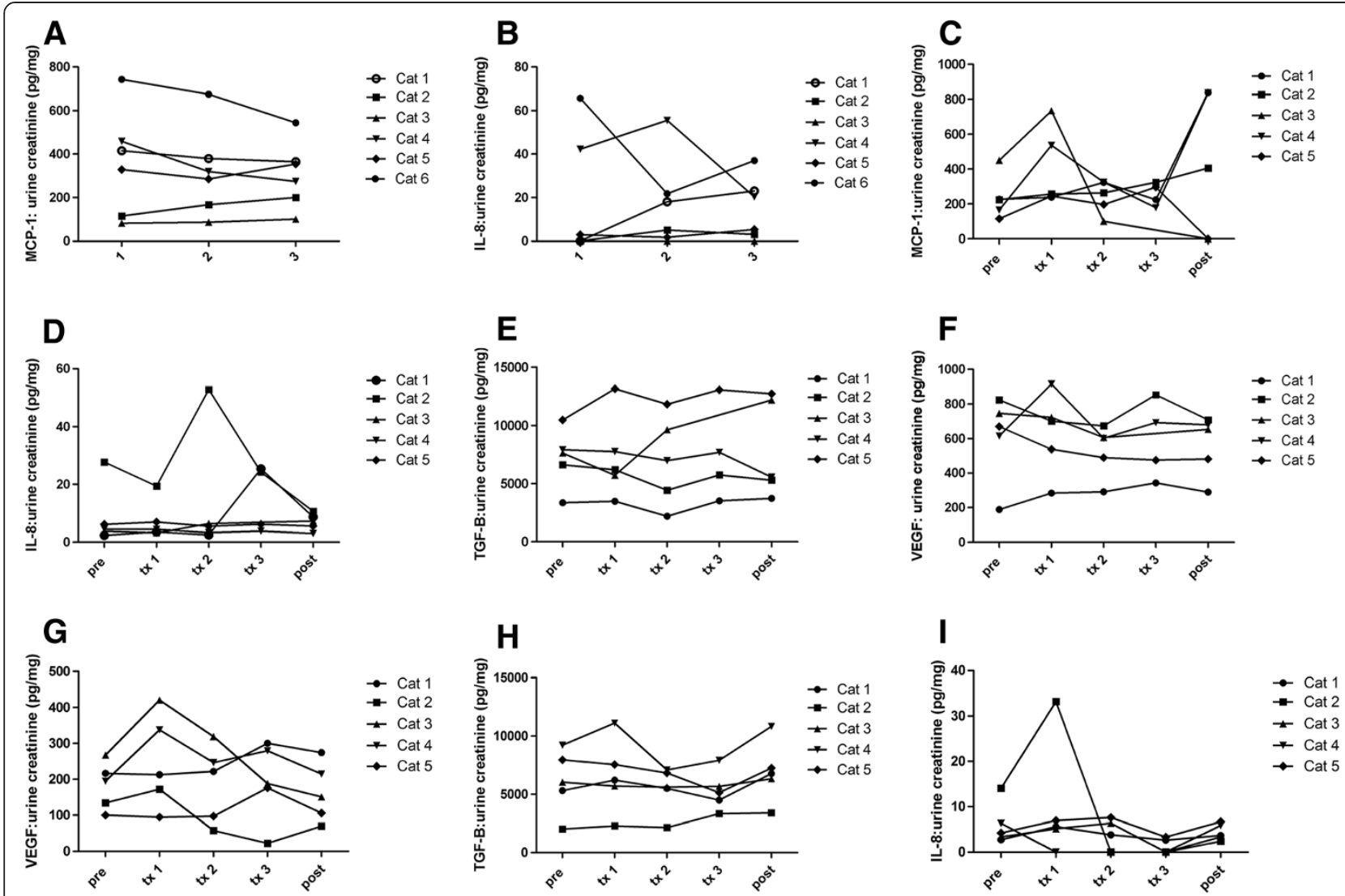

Figure 4 Urinary cytokine levels in mesenchymal stem cell (MSC)-treated cats. Pilot study 1 (A,B): cats received $2 \times 10^{6}$ cryopreserved aMSCs intravenously every other week for three injections. (A) Monocyte chemoattractant protein 1 (MCP-1):urine creatinine ratio throughout the study. There was a statistically significant decrease in MCP-1 $(P=0.0001)$. (B) Interleukin 8 (IL-8):urine creatinine ratio throughout the study. There was a statistically significant decrease in IL-8 $(P=0.01)$. Pilot study $2(C-E)$ : cats received $4 \times 10^{6}$ cryopreserved aMSCs intravenously every other week for three injections. Cytokine:urine creatinine ratio data throughout the study for (C) MCP-1, (D) IL-8, (E) transforming growth factor (TGF)- $\beta 1$ and (F) vascular endothelial growth factor (VEGF). There was no statistically significant change in urinary cytokines in pilot study 2 . Pilot study 3 (G-I): cats received $4 \times 10^{6}$ aMSCs cultured from cryopreserved adipose intravenously every other week for three injections. Cytokine:urine creatinine ratio data throughout the study for (G) IL-8, (H) TGF- $\beta 1$ and (I) VEGF. There was no statistically significant change in urinary cytokines in pilot study 3 .

adverse effects from aMSC administration. However, the degree of this decrease $(<0.5 \mathrm{mg} / \mathrm{dl})$ is very small and may not translate to any change in clinical disease. In contrast, higher intravenous doses of cryopreserved aMSCs were associated with a high incidence of adverse effects and variable evidence of improvement in renal function as evidenced by GFR estimated by iohexol clearance. Most of the five cats in pilot study 2 that received $4 \times 10^{6}$ cryopreserved aMSCs per injection experienced side effects, including vomiting during infusion and increased respiratory rate and effort including overt dyspnea in one case. Significant changes in serum creatinine, GFR, and urinary cytokine concentrations were not observed in pilot study 2 cats. In pilot study 3 , cats that received $4 \times 10^{6}$ aMSCs cultured from cryopreserved adipose experienced no side effects, but little evidence of clinically significant improvement in renal function. Thus, we concluded that although intravenous administration of higher doses of cryopreserved, allogeneic aMSCs was unlikely to be an acceptable treatment option due to the high rate of complications and the lack of clinical or experimental responses; aMSCs expanded from cryopreserved fat appear a safer option although strong indication of efficacy is still lacking.

A recent study by Semedo et al. [4] in a rat model of CKD provided compelling evidence of the potential efficacy of repeated intravenous administration of bone marrowderived MSCs for suppression of intrarenal inflammation and fibrosis, and this treatment protocol was the one we adapted for clinical application in CKD cats. Although aMSCs were used instead of bone marrow-derived MSCs, in the field of adipose stem cell research, the two sources are felt to be comparable in characterization and therapeutic potential in inflammatory disease states [23-27,32,33]. Why then were the results of our preliminary studies of aMSC therapy in cats with CKD so different 
from the rodent studies, when similar doses of MSCs (based on body weight) were utilized? Firstly, differences in the CKD model should be taken into consideration. Most rodent models involve acute insult to the kidney with subsequent administration of MSCs within a few weeks: a relatively short time post renal insult $[4,34]$. Thus, the induced disease models, with their relatively short time frame, may not be representative of the changes occurring in a truly chronic, naturally occurring disease process [11]. Cats have an extended lifespan and often have CKD for months to years prior to clinical diagnosis and study enrollment, which is more similar to the disease time frame seen in humans. The possibility that this potential therapy may not be as effective in patients with more long-standing disease should be considered.

Secondly, in our study, we utilized allogeneic aMSCs, whereas autologous MSCs were used in the Semedo et al. study [4]. The relative efficacy of autologous vs. allogeneic cells is an area of controversy. Although allogeneic MSCs are immune privileged and are not expected to incite an immune response, according to some authors they may not be as effective as autologous cells [35]. It is argued that autologous MSCs may survive longer in the body in comparison to allogeneic cells, which could reduce efficacy of the latter. Decreased efficacy of allogeneic MSCs in comparison to autologous MSCs has been observed in one acute renal failure rodent study [35]. However, allogeneic MSCs have been widely used in experimental stem cell transfer investigations, including clinical trials in humans, with positive results [35,36].

A third major difference was the use of freshly-thawed, cryopreserved aMSCs in pilot studies 1 and 2 . This decision was made based on the logistical ease afforded by use of cryopreserved rather than freshly-cultured MSCs. Successful cryopreservation of cells has been previously described [37]. However, it is unknown if cryopreservation affects the functional properties of aMSCs necessary for successful use in this model. Previous studies assessing the effects of cryopreservation on MSCs did not examine effects on potential immunogenicity and further work is needed to fully assess this subject $[37,38]$.

Based on the results of the three pilot studies, it appears that use of higher doses of cryopreserved aMSCs was the source of the treatment-related adverse effects in pilot study 2 as similar doses of aMSCs cultured from cryopreserved adipose tissue did not result in any adverse effects. Although the specific reasons for the increased incidence of side effects is not known, it is likely related to the increase in dose of cryopreserved cells. The most likely explanation for this reaction is an instant blood mediated inflammatory reaction (IBMIR) which results in clumping of the cells as they contact the blood and potential subsequent micro pulmonary thromboembolism [39]. The IBMIR phenomenon has been described previously in cryopreserved cells and increases in severity with dose and passage number [39]. It can result in lysis of the administered MSCs and subsequent poor efficacy. Although all cells given in pilot study 2 were of the same passage (P3) as those used in the other two pilot studies, the reaction was only seen in the pilot study group where cells were taken directly from cryopreservation and used at the higher dose. In pilot study 3 no complications during or after administration of aMSCs cultured from cryopreserved fat were appreciated. Thus, we have concluded that the administration of a higher dose of aMSCs taken directly from cryopreservation, despite careful washing, was the source of the toxic reactions observed, and this form of administration is not recommended.

Although several cats in the pilot study 1 group experienced a statistically significant improvement in serum creatinine concentrations during the study, the degree of increase is unlikely to be clinically significant. Creatinine is not the most sensitive measurement of renal function even though it is the routinely measured clinical marker of renal function. Several factors that are not directly related to renal function can influence serum creatinine values, including muscle mass and hydration status $[28,40]$. Decreases in weight and/or muscle mass can potentially decrease serum creatinine, while a worsening in hydration status can increase serum creatinine. In addition, changes to renal function may occur without any concomitant alteration in serum creatinine, although this is thought to be less true once an animal reaches the stage of moderate renal dysfunction [28]. Although there were no significant changes in body weight noted in these pilot studies, individual changes in body weight in the study cats could potentially have confounded serum creatinine measurements, while changes in bladder fullness at the time of weighing could potentially have compromised weight values. Therefore, in cats in pilot studies 2 and 3 we also measured estimated GFR, as a more objective way to monitor changes in renal function. Quantitating GFR allowed us to assess potential overall kidney function changes induced by the paracrine and/or autocrine effects of MSC administration. GFR results were variable in cats in pilot studies 2 and 3 ; two of five animals in pilot study 1 and two of five cats in pilot study 3 did experience a marked increase from pretreatment baseline; however the change in GFR was not significant overall for the group. Overall, definitive evidence of significant efficacy of intravenous administration of allogeneic aMSCs for treatment of CKD in cats is lacking at this time. Closer evaluation of the mechanism of MSC action in CKD, effect of disease stage, necessary cell dosage (for example, a cell/kg dose rather than a per cat dose as used in these pilot studies), timing of injections, and other variables remain to be determined. 


\section{Conclusions}

The safety and potential efficacy of repeated intravenous administration of allogeneic cryopreserved feline aMSCs to cats with naturally occurring CKD was assessed in this preliminary study. The major findings from our pilot studies of aMSC therapy in cats with CKD were that lower doses of allogeneic cryopreserved aMSCs were well tolerated following repeated intravenous administration and appeared to be associated with modest decrease in serum creatinine. In contrast, higher intravenous doses of cryopreserved aMSCs were associated with a high incidence of adverse effects and only modest evidence of functional improvement in renal function. Thus, we concluded that intravenous administration of higher doses of cryopreserved, allogeneic aMSCs was unlikely to be an acceptable treatment option due to the high rate of complications and the lack of clinical or experimental responses.

\section{Abbreviations \\ aMSCs: Adipose-derived mesenchymal stem cells; CBC: Complete blood count; CKD: Chronic kidney disease; GFR: Glomerular filtration rate; HBSS: Hank's balanced salt solution; IBMIR: Instant blood mediated inflammatory reaction; IL: interleukin; MCP-1: Monocyte chemoattractant protein 1; MSCs: Mesenchymal stem cells; SPF: Specific pathogen-free; TGF- $\beta 1$ : Transforming growth factor- $\beta 1$; UPC: Urine protein-creatinine ratio; VEGF: Vascular endothelial growth factor.}

\section{Competing interests}

The authors declare they have no competing interests.

\section{Authors' contributions}

JMQ conceived and designed the study, harvested adipose tissue, prepared injections, provided veterinary care and administered treatments to enrolled animals, performed data acquisition, analysis and manuscript writing and revision. TLW performed the isolation and expansion of MSCs, urine cytokine analysis, flow cytometry and differentiation assays, prepared injections, and manuscript writing and revision. LAH performed the sample preparation, data entry, and urine cytokine analysis, and manuscript writing. The study was conducted in the laboratory of SWD and he was responsible for supervision of the study and manuscript writing and revision. All authors read and approved the final manuscript.

\section{Acknowledgements}

The authors would like to acknowledge Dr Sue Vandewoude for providing adipose tissues from SPF cats for use in this study. This work was supported by the Morris Animal Foundation, Frankie's Fund for Feline Stem Cell Research and by a grant from Bohemian Foundation. This work was also supported in part by Award Number K08Al071724, awarded to TLW, from the National Institute of Allergy and Infectious Diseases. The content is solely the responsibility of the authors and does not necessarily represent the official views of the National Institute of Allergy and Infectious Diseases or the National Institutes of Health.

\section{Author details}

${ }^{1}$ Department of Clinical Sciences, Immunology, and Pathology, Center for Immune and Regenerative Medicine, College of Veterinary Medicine and Biomedical Sciences, Colorado State University, Fort Collins, CO 80523, USA. ${ }^{2}$ Department of Microbiology, Immunology, and Pathology, Center for Immune and Regenerative Medicine, College of Veterinary Medicine and Biomedical Sciences, Colorado State University, Fort Collins, CO 80523, USA.

Received: 9 August 2012 Revised: 14 March 2013

Accepted: 23 April 2013 Published: 30 April 2013

\section{References}

1. DiBartola SP, Rutgers HC, Zack PM, Tarr MJ: Clinicopathologic findings associated with chronic renal disease in cats: 74 cases (1973-1984). J Am Vet Med Assoc 1987, 190:1196-1202.

2. Chakrabarti S, Syme HM, Brown CA, Elliott J: Histomorphometry of feline chronic kidney disease and correlation with markers of renal dysfunction. Vet Pathol 2013, 50:147-155.

3. Lee SR, Lee SH, Moon JY, Park JY, Lee D, Lim SJ, Jeong KH, Park JK, Lee TW, Ihm CG: Repeated administration of bone marrow-derived mesenchymal stem cells improved the protective effects on a remnant kidney model. Ren Fail 2010, 32:840-848.

4. Semedo P, Correa-Costa M, Antonio Cenedeze M, Maria- Avancini-Costa -Malheiros D, Antonia-dos-Reis M, Shimizu MH, Seguro AC, Pacheco-Silva A, Saraiva-Camara NO: Mesenchymal stem cells attenuate renal fibrosis through immune modulation and remodeling properties in a rat remnant kidney model. Stem Cells 2009, 27:3063-3073.

5. Villanueva S, Ewertz E, Carrión F, Tapia A, Vergara C, Céspedes C, Sáez PJ, Luz P, Irarrázabal C, Carreño JE, Figueroa F, Vio CP: Mesenchymal stem cell injection ameliorates chronic renal failure in a rat model. Clin Sci (Lond) 2011, 121:489-499.

6. Ninichuk V, Gross O, Segerer S, Hoffmann R, Radomska E, Buchstaller A, Huss $\mathrm{R}$, Akis $\mathrm{N}$, Schlondorff $\mathrm{D}$, Anders $\mathrm{HJ}$ : Multipotent mesenchymal stem cells reduce interstitial fibrosis but do not delay progression of chronic kidney disease in collagen4A3-deficient mice. Kidney Int 2006, 70:121-129.

7. Cavaglieri RC, Martini D, Sogayar MC, Noronha IL: Mesenchymal stem cells delivered at the subcapsule of the kidney ameliorate renal disease in the rat remnant kidney model. Transplant Proc 2009, 41:947-951.

8. Morigi M, Imberti B, Zoja C, Corna D, Tomasoni S, Abbate M, Rottoli D, Angioletti S, Benigni A, Perico N, Alison M, Remuzzi G: Mesenchymal stem cells are renotropic, helping to repair the kidney and improve function in acute renal failure. J Am Soc Nephrol 2004, 15:1794-1804.

9. Kitamura S, Yamasaki Y, Kinomura M, Sugaya T, Sugiyama $H$, Maeshima $Y$ Makino $\mathrm{H}$ : Establishment and characterization of renal progenitor like cells from S3 segment of nephron in rat adult kidney. FASEB J 2005, 19:1789-1797

10. Kim SS, Park HJ, Han J, Gwak SJ, Park MH, Song KW, Rhee YH, Min-Chung H, Kim BS: Improvement of kidney failure with fetal kidney precursor cell transplantation. Transplantation 2007, 83:1249-1258.

11. Little MH, Rae FK: Review article: potential cellular therapies for renal disease: can we translate results from animal studies to the human condition? Nephrology (Carlton) 2009, 14:544-553.

12. Chhabra P, Brayman KL: The use of stem cells in kidney disease. Curr Opin Organ Transplant 2009, 14:72-78.

13. Choi SJ, Kim JK, Hwang SD: Mesenchymal stem cell therapy for chronic renal failure. Expert Opin Biol Ther 2010, 10:1217-1226.

14. Asanuma H, Meldrum DR, Meldrum KK: Therapeutic applications of mesenchymal stem cells to repair kidney injury. J Urol 2010, 184:26-33.

15. O'Brien T, Barry FP: Stem cell therapy and regenerative medicine. Mayo Clin Proc 2009 , 84:859-861.

16. Zerbini G, Piemonti L, Maestroni A, Dell'Antonio G, Bianchi G: Stem cells and the kidney: a new therapeutic tool? J Am Soc Nephrol 2006 17:S123-126.

17. Zubko R, Frishman W: Stem cell therapy for the kidney? Am J Ther 2009, 16:247-256

18. Reinders ME, Fibbe WE, Rabelink TJ: Multipotent mesenchymal stromal cell therapy in renal disease and kidney transplantation. Nephrol Dial Transplant 2010, 25:17-24.

19. Deak E, Ruster B, Keller L, Eckert K, Fichtner I, Seifried E, Henschler R: Suspension medium influences interaction of mesenchymal stromal cells with endothelium and pulmonary toxicity after transplantation in mice. Cytotherapy 2010, 12:260-264.

20. Togel F, Hu Z, Weiss K, Isaac J, Lange C, Westenfelder C: Administered mesenchymal stem cells protect against ischemic acute renal failure through differentiation-independent mechanisms. Am J Physiol Renal Physiol 2005, 289:F31-42.

21. Quimby JM, Webb TL, Gibbons DS, Dow SW: Evaluation of intrarenal mesenchymal stem cell injection for treatment of chronic kidney disease in cats: a pilot study. J Feline Med Surg 2011, 13:418-426.

22. Webb TL, Quimby JM, Dow SW: In vitro comparison of feline bone marrow-derived and adipose tissue-derived mesenchymal stem cells. J Feline Med Surg 2012, 14:165-168. 
23. Minteer D, Marra KG, Rubin JP: Adipose-derived mesenchymal stem cells: biology and potential applications. Adv Biochem Eng Biotechnol 2013, 129:59-71.

24. Castelo-Branco MT, Soares ID, Lopes DV, Buongusto F, Martinusso CA, doRosario A, Souza SA, Gutfilen B, Fonseca LM, Elia C, Madi K, Schanaider A, Rossi MI, Souza HS: Intraperitoneal but not intravenous cryopreserved mesenchymal stromal cells home to the inflamed colon and ameliorate experimental colitis. PLoS One 2012, 7:e33360

25. Puissant B, Barreau C, Bourin P, Clavel C, Corre J, Bousquet C, Taureau C, Cousin B, Abbal M, Laharrague P, Penicaud L, Casteilla L, Blancher A: Immunomodulatory effect of human adipose tissue-derived adult stem cells: comparison with bone marrow mesenchymal stem cells. $\mathrm{Br} J$ Haematol 2005, 129:118-129.

26. Yanez R, Lamana ML, Garcia-Castro J, Colmenero I, Ramirez M, Bueren JA: Adipose tissue-derived mesenchymal stem cells have in vivo immunosuppressive properties applicable for the control of the graftversus-host disease. Stem Cells 2006, 24:2582-2591.

27. Shin S, Kim Y, Jeong S, Hong S, Kim I, Lee W, Choi S: The therapeutic effect of human adult stem cells derived from adipose tissue in endotoxemic rat model. Int J Med Sci 2013, 10:8-18.

28. Kerl ME, Cook CR: Glomerular filtration rate and renal scintigraphy. Clin Tech Small an P 2005, 20:31-38.

29. Miyamoto K: Use of plasma clearance of iohexol for estimating glomerular filtration rate in cats. Am J Vet Res 2001, 62:572-575.

30. Le Garreres A, Laroute V, De La Farge F, Boudet KG, Lefebvre HP: Disposition of plasma creatinine in non-azotaemic and moderately azotaemic cats. J Feline Med Surg 2007, 9:89-96.

31. Habenicht LM, Webb TL, Clauss LA, Dow SW, Quimby JM: Urinary cytokine levels in apparently healthy cats and cats with chronic kidney disease. J Feline Med Surg 2013, 15:99-104

32. Gao J, Liu R, Wu J, Liu Z, Li J, Zhou J, Hao T, Wang Y, Du Z, Duan C, Wang $C$ : The use of chitosan based hydrogel for enhancing the therapeutic benefits of adipose-derived MSCs for acute kidney injury. Biomaterials 2012, 33:3673-3681.

33. Furuichi K, Shintani H, Sakai Y, Ochiya T, Matsushima K, Kaneko S, Wada T: Effects of adipose-derived mesenchymal cells on ischemia-reperfusion injury in kidney. Clin Exp Nephrol 2012, 16:679-689.

34. Choi S, Park M, Kim J, Hwang S, Park S, Lee Y: The role of mesenchymal stem cells in the functional improvement of chronic renal failure. Stem Cells Dev 2009, 18:521-529.

35. Togel F, Zhang P, Hu Z, Westenfelder C: VEGF is a mediator of the renoprotective effects of multipotent marrow stromal cells in acute kidney injury. J Cell Mol Med 2009, 13:2109-2114.

36. McTaggart SJ, Atkinson K: Mesenchymal stem cells: immunobiology and therapeutic potential in kidney disease. Nephrology (Carlton) 2007, 12:44-52.

37. Martinello T, Bronzini I, Maccatrozzo L, Mollo A, Sampaolesi M, Mascarello F, Decaminada M, Patruno M: Canine adipose-derived-mesenchymal stem cells do not lose stem features after a long-term cryopreservation. Res Vet Sci 2011, 91:18-24.

38. Ginis I, Grinblat B, Shirvan MH: Evaluation of bone marrow-derived mesenchymal stem cells after cryopreservation and hypothermic storage in clinically safe medium. Tissue Eng Part C Methods 2012, 6:453-463.

39. Moll G, Rasmusson-Duprez I, von Bahr L, Connolly-Andersen AM, Elgue G, Funke L, Hamad OA, Lonnies H, Magnusson PU, Sanchez J, Teramura Y, Nilsson-Ekdahl K, Ringden O, Korsgren O, Nilsson B, Le Blanc K: Are therapeutic human mesenchymal stromal cells compatible with human blood? Stem Cells 2012, 30:1565-1574.

40. Baxmann AC, Ahmed MS, Marques NC, Menon VB, Pereira AB, Kirsztajn GM Heilberg IP: Influence of muscle mass and physical activity on serum and urinary creatinine and serum cystatin C. Clin J Am Soc Nephrol 2008, 3:348-354.

doi:10.1186/scrt198

Cite this article as: Quimby et al:: Safety and efficacy of intravenous infusion of allogeneic cryopreserved mesenchymal stem cells for treatment of chronic kidney disease in cats: results of three sequential pilot studies. Stem Cell Research \& Therapy 2013 4:48.

\section{Submit your next manuscript to BioMed Central and take full advantage of:}

- Convenient online submission

- Thorough peer review

- No space constraints or color figure charges

- Immediate publication on acceptance

- Inclusion in PubMed, CAS, Scopus and Google Scholar

- Research which is freely available for redistribution 